\title{
A new approach for parameters of Nucleon-Nucleon Scattering at low energies in one and two dimensions
}

\author{
Shojaei, Mohammad Reza; Amini, Maryam; Zoghi-Foumani, Niloufar*
}

Department of Physics, Shahrood University of Technology, P. O. Box 36155-316, Shahrood, Iran

Keywords: Scattering cross section, Nikiforov-Uvarov (NU) method, WKB approximation

\begin{abstract}
Many of the important properties of multi-nucleon systems can be found by studying the nucleon- nucleon interactions. In this work, a suitable central and an appropriate noncentral potential have been used to study nucleon- nucleon scattering in one and two dimensions. The phase shift, scattering cross section, length and amplitude are calculated using the Nikiforov-Uvarov (NU) method and WKB approximation. Comparison between the results of the suggested model in this article and the experimental reported values indicates that there is a good consistency between them.
\end{abstract}

\section{Introduction}

One of the main challenges of nuclear physics is to find the static and dynamic properties of nuclei and interpret the interactions between nucleons. To the best of our knowledge, a helpful method to determine the structure of nucleus is to use scattering phenomenon. It leads to the exploration of nuclear forces and the laws governing the interactions of elementary particles [1and 2]. In addition, scattering can be used to specify the characteristics of the interactions between nucleons [3and 4]. One ideal approach is to discuss dispersion equations formulated for neutronproton scattering [5and 6]. When an incoming particle (which is described with a wave packet) closes to the target and creates an interaction, there are two wave packets as output: One of them is toward the direct route, which describes the section of the beam which is not dispersed, the other one is deflected by an angle which is related to the dispersed particles. The Number of dispersed particles in a given time and space angle of the incoming flux is introduced as the differential cross section [6].

Scattering is an appropriate facility to study the nuclear properties which is continuing as an active area of research [7]. In this paper, a comprehensive description of quantum scattering in one dimension in close analogy with the two- and three-dimensional cases [4 and 8] is presented. Apart from these interests in research, the study of one-dimensional scattering is also advantageous from a pedagogical point of view [9]. In a one dimensional treatment for simple potentials such as the potential well special mathematical functions such as the Bessel's functions are not needed, while it still has sufficient complicacy to illustrate many of the physical processes which occur in two and three dimensions [10,11 and 12]. Hence, the presented discussion about the one dimensional scattering is expected to assist the understanding of the more complex scattering problems namely two and three dimensional scattering [2]. The quantum mechanical scattering problem in two dimensions (2D) has been discussed widely in Refs. [1, 2 and 8]. The inherent circular symmetry in 2D renders the free partial-wave radial function to be the ordinary Bessel (regular at the origin) and Neumann (irregular) functions, in contradistinction to the three-dimensional (3D) case where these functions become the spherical counterpart (spherical Bessel and Neumann functions) [11 and 12]. Besides being an interesting pedagogic topic at the graduate level, 2D scattering has applications in surface physics [2]. It has also important applications in atomic, molecular and nuclear scattering where, at high energies, one usually encounters sums over prohibitively large numbers of partial waves [6 and 13]. Such sums are converted into integrals and efficiently evaluated in semi- classical scattering [14 and 12]. The concepts employed in the field of wave optics found a very natural adaptation in semi- classical 3D scattering, where one deals with the optics of matter waves. As long as energy $E \gg|V|$, it is not needed for the potential $V$ to be weak [15]. Under thesesituations the semi- classical path concept is valid and the quantum wave-function is replaced by the semi- 
classical Wentzel-Kramers-Brillouin (WKB) wave function [11 and 16]. The results for 2D scattering in the WKB approximation [6] are presented as well as comprehensive discussion of rainbow and glory scattering and orbiting.

In this article, quantum scattering in one-dimension is studied by analytically solving the Schrödinger equation with Woods-Saxon potential. The phase shift, Scattering cross section, length and amplitude in one dimension is obtained at various low energies. Then, quantum scattering in two-dimensions is presented and the WKB approximation is used to calculate the phase shift, Scattering cross section, length and amplitude in two dimensions. The calculated results are compared with the experimental ones and it is found that there is a reasonable consistency between them.

\section{Quantum scattering in one dimension}

The time-independent Schrödinger equation which describes scattering for the reduced mass of $\mu$, potential of $\mathrm{V}(\mathrm{r})$ and energy of $E$ is as follow [10 and 14]:

$$
\left[-\frac{\hbar^{2}}{2 \mu} \nabla^{2}+V(r)\right] \psi(r)=E \psi(r)
$$

Solving this equation with a specific potential yields the energy eigenvalues which can be put in the following equation to obtain the phase shift:

$$
\delta=\tan ^{-1}\left(\frac{k}{k_{2}} \tan k_{2} a\right)-k a
$$

Where $k^{2}=\frac{2 \mu}{\hbar^{2}} E, k_{2}{ }^{2}=\frac{2 \mu}{\hbar^{2}}\left(E+V_{0}\right)$ and $a$ is the potential width [1, 17].

In order to calculate the scattering amplitude $f$, the phase shift and the equation bellow are considered [1, 18 and 19]:

$$
f=\frac{\exp (i \delta)}{k} \sin \delta
$$

Using the following equation, the total cross section can be calculated for various values of the phase shift [1, 10 and 20]:

$$
\sigma=\frac{4 \pi}{k^{2}} \sum_{l=0}^{\infty}(2 l+1) \sin ^{2} \delta
$$

Where $l$ represents the angular quantum number. The total cross section is equal to the probability of scattering in all directions as follow:

$$
\sigma_{\text {tot }}(k)=\int d \Omega \frac{d \sigma}{d \Omega}
$$

\section{Scattering in one dimension in the presence of Woods-Saxon potential}

Choosing a suitable potential for the interactions between nucleons plays a crucial role in calculations purposed in this paper. The Woods-Saxon potential which is one of the most important and acceptable central potentials in nuclear physics is defined as [21]:

$$
V(r)=\frac{-V_{0}}{1+\exp \left(\frac{r-R_{0}}{a}\right)}
$$


Where $V_{o}, R_{o}$ and $a$ are the potential depth, width and its surface diffuseness, respectively. $V_{o}$ is usually adjusted to the experimental values of ionization energies. After obtaining the energy eigenvalues, the phase shift, scattering cross section, amplitude and length can be calculated for the neutron-proton scattering in the presence of Woods-Saxon potential. At low energies, scattering occurs mostly in state of $S$, so that one can focus on the radial quantum number of $l=0$ [14]. To solve the Schrödinger equation with Woods-Saxon potential and obtain the energy eigenvalues the NU method is used which is briefly described in the next part.

\subsection{Nikiforov-Uvarov Method}

NU method is based on the solution of a general second order linear differential equation with special orthogonal functions and a non-relativistic Schrödinger equation can be solved with this method. For a given real and complex potential, the Schrödinger equation is reduced to a generalized equation of hypergeometric type with an appropriate coordinate transformation $s=s(r)$

Therefore it can be written in the following form [22 and 23]:

$$
\psi^{\prime \prime}(s)+\frac{\tilde{\tau}(s)}{\sigma(s)} \psi_{n}^{\prime}(s)+\frac{\tilde{\sigma}(s)}{\sigma^{2}(s)} \psi_{n}(s)=0,
$$

Where $\sigma$ and $\tilde{\sigma}$ are polynomials (at most second-degree) and is a first-degree polynomial. By using the transformation:

$$
\psi(s)=\varphi(s) y(s)
$$

equation (7) reduces to a hypergeometric-type form as follow:

$$
\sigma(s) y_{n}^{\prime \prime}(s)+\tau(s) y_{n}^{\prime}(s)+\lambda y_{n}(s)=0
$$

Where the function $\varphi(s)$ is defined as a logarithmic derivative:

$$
\sigma(s)=\pi(s) \frac{\phi(s)}{\phi^{\prime}(s)}
$$

$y_{n}(s)$ is the hypergeometric type function whose polynomial solutions are given by Rodrigues relation:

$$
y_{n}(s)=\frac{B_{n}}{\rho_{n}} \frac{d^{n}}{d s^{n}}\left(\sigma^{n}(s) \rho(s)\right)
$$

Where $B_{n}$ is a normalizing constant and the weight function $\rho$ must satisfy the condition:

$$
(\sigma \rho)^{\prime}=\tau \rho
$$

The function $\pi(s)$ and the parameter $\lambda$ required for this method are defined as:

$$
\pi(s)=\frac{\sigma^{\prime}(s)-\tilde{\tau}(s)}{2} \pm \sqrt{\left(\frac{\sigma^{\prime}(s)-\tilde{\tau}(s)}{2}\right)^{2}-\tilde{\sigma}(s)+K \sigma(s)}, \quad \lambda=K+\pi^{\prime}(s)
$$

On the other hand, in order to find the value of $K$, the expression under the square root must be square of a polynomial. Thus, a new eigenvalue equation for the Schrödinger equation yields the relation bellow:

$$
\lambda=\lambda_{n}=-n \tau^{\prime}(s)-\frac{n(n-1)}{2} \sigma^{\prime \prime}(s) \quad(n=0,1,2, \ldots \ldots)
$$


Where

$$
\tau(s)=\tilde{\tau}(s)+2 \pi(s) .
$$

It must have a negative derivative [22 and 23].

\subsection{Energy eigenvalues of Woods- Saxon Potential}

In order to calculate the parameters of scattering, the energy eigenvalues of Schrödinger equation have to be obtained. The considered central potential for the interactions between nucleons in this paper is the Woods-Saxon potential. The radial part of time-independent Schrödinger equation with Woods-Saxon potential takes the form [24]:

$$
\frac{d^{2} \psi(r)}{d r^{2}}+\frac{2 \mu}{\hbar^{2}}\left[E+\frac{V_{0}}{1+e^{\left(r-R_{0}\right) / a}}\right] \psi(r)+\frac{\ell(\ell+1)}{r^{2}} \psi(r)=0
$$

where the radial wave function $\psi(r)$ is written as $\psi(r)=R(r) / r$ and $r-R_{0} \equiv r$. In addition, here it is assumed that $\psi(r)$ is bounded as $r \rightarrow 0$. Solving this equation is not a simple job, in order to get a form that NU method is applicable the transformations mentioned bellow can be helpful:

$$
\begin{gathered}
\left\{\begin{array}{l}
\ell(\ell+1) \equiv \gamma \\
1 / a \equiv 2 \alpha
\end{array}\right. \\
\frac{1}{r^{2}} \approx \frac{1}{\alpha^{2}} \frac{e^{-2 \alpha r}}{1-e^{2 \alpha r}}
\end{gathered}
$$

With $s=c e^{2 a r-1}$ the equation (16) leads to the generalized form of hypergeometric type given in equation (7):

$$
\frac{d^{2} R(s)}{d s^{2}}+\frac{s}{s(c+s)} \frac{d R(s)}{d s}+\frac{1}{s^{2}(c+s)^{2}} \times\left[\left(\varepsilon^{2}+\Omega^{2}+\eta_{1}^{2}-\eta_{4}^{2}\right) s^{2}+\left(\beta+\eta_{3}^{2}\right) s+\eta_{2}^{2}\right] R(s)=0
$$

where

$$
\begin{aligned}
c & =e^{-2 \alpha R_{0}} \\
\varepsilon^{2} & =-\frac{\mu E}{2 \hbar^{2} \alpha^{2}} \\
\beta^{2} & =\frac{\mu V_{0}}{2 \hbar^{2} \alpha^{2}(1+c)} \\
\Omega^{2} & =\frac{\mu V_{0}}{2 \hbar^{2} \alpha^{2}(1+c)^{2}}
\end{aligned} \quad\left\{\begin{array}{l}
\eta_{1}^{2}=\frac{1}{\alpha^{4}} \gamma \\
\eta_{2}^{2}=\frac{c^{2}}{\alpha^{4}} \gamma \\
\eta_{3}^{2}=\frac{2 c}{\alpha^{4}} \gamma \\
\eta_{4}^{2}=\frac{\mu}{2 \hbar^{2} \alpha^{2}}
\end{array}\right.
$$

Comparing equation (19) with equation (7) yields the corresponding polynomials as follows:

$$
\begin{aligned}
& \tilde{\tau}(s)=s \\
& \sigma(s)=s(c+s) \\
& \tilde{\sigma}(s)=-\left(\varepsilon^{2}+\Omega^{2}+\eta_{1}^{2}+\eta_{4}^{2}\right) s^{2}+\left(\beta^{2}+\eta_{3}^{2}\right) s+\eta_{2}^{2}
\end{aligned}
$$

Substituting these polynomials in equation $(13), \pi(s)$ can be achieved as:

$$
\pi(s)=\frac{c+s}{2} \pm \frac{1}{2} \sqrt{\left(4 K-b_{1}\right) s^{2}+\left(b_{2}+4 c K\right) s+b_{3}}
$$

with

$$
b_{1}=-4 a+1 \quad b_{2}=2 c-4 b \quad b_{3}=c^{2}-4 d
$$


and

$$
a=\varepsilon^{2}+\Omega^{2}+\eta_{1}^{2}+\eta_{4}^{2} \quad b=\beta^{2}+\eta_{3}^{2} \quad d=\eta_{2}^{2}
$$

The constant $K$ is determined according to the rule that the expression under the square root must be square of a polynomial and since $\tau(s)$ in equation (15) must have a negative derivative, the most appropriate answer for $\pi(s)$ i:

$$
\pi(s)=\frac{c+s}{2}-\frac{1}{2 c^{2}}\left[\sqrt{b_{2} s}-\sqrt{(1-c) \mid b_{2}^{2}+b_{1} c^{2}}\right]
$$

And finally the energy eigenvalues are obtained as follow:

$$
\begin{aligned}
E_{n}= & 1+\frac{2 \hbar^{2} \gamma}{\mu \alpha^{2}}+\frac{V_{0}}{(1+c)^{2}}-\left(\frac{\hbar^{2}}{2 \mu}\right) \alpha^{2}-\left(\frac{\hbar^{2}}{8 \mu}\right)^{2} \alpha^{2}\left[2 c-\left(\frac{4 \mu}{\hbar^{2} \alpha^{2}}\right)\left(\frac{V_{0}}{1+c}+c\left(\frac{2 \hbar^{2}}{\mu}\right)\left(\frac{\gamma}{\alpha^{2}}\right)\right)\right](c-1) \\
& \left.-\frac{2 \hbar^{2}}{\mu} \alpha^{2}\left[\frac{2 n}{n \sqrt{2 c-\frac{2 \mu}{\hbar^{2} \alpha^{2}}}\left(\frac{V_{0}}{1+c}+c\left(\frac{2 \hbar^{2} \gamma}{\mu \alpha^{2}}\right)\right)}+\frac{4+c-3 n(n-1)}{2\left[2 c-\frac{2 \mu}{\hbar^{2} \alpha^{2}}\left(\frac{V_{0}}{1+c}+c\left(\frac{2 \hbar^{2} \gamma}{\mu \alpha^{2}}\right)\right)\right]}-\frac{1}{c}\right]^{2}\right]
\end{aligned}
$$

This equation is used to obtain the scattering phase shift, amplitude and cross section using the equations (2), (3), (4) and (5) mentioned before in sec 2.

\subsection{Scattering cross section, length and amplitude in one dimension}

Substituting the energy equation (26) in the equations related to scattering in one dimension, the phase shift, scattering cross section and amplitude for various values of the potential depth are calculated. In addition, the scattering length which describes low- energy scattering in quantum mechanics is obtained using the equation bellow [1 and 2]:

$$
a_{0}=-\lim _{k \rightarrow 0} \frac{1}{k} \tan \delta_{0}
$$

Some of the results are listed in the following table which are nearly reasonably consistent with the experimental data.

Table1: Scattering cross section, length and amplitude in the presence of Woods-Saxon potential (with $n=1, R=5.14 \mathrm{fm}, a=0.65 \mathrm{fm}$ )

\begin{tabular}{|l|l|l|l|l|}
\hline$V_{0}(\mathrm{MeV})$ & \multicolumn{1}{|c|}{$E(\mathrm{MeV})$} & $f(\mathrm{fm})$ & \multicolumn{1}{|c|}{$\sigma_{\text {tot }}(b)$} & \multicolumn{1}{|c|}{$a_{0}(\mathrm{fm})$} \\
\hline 6.06 & 5.41 & 2.95 & 13.26 & 36.80 \\
\hline 6.55 & 5.38 & 2.58 & 15.01 & 38.62 \\
\hline 6.96 & 5.14 & 2.31 & 15.58 & 39.12 \\
\hline 6.44 & 4.34 & 2.28 & 19.87 & 43.41 \\
\hline 6.39 & 4.14 & 2.01 & 24.21 & 47.75 \\
\hline 6.70 & 4.05 & 2.15 & 24.69 & 48.23 \\
\hline 7.73 & 4.00 & 2.01 & 26.87 & 50.41 \\
\hline
\end{tabular}

According to the experimental reports, the scattering cross section at low energies has a nearly constant value between $4 b$ and $5 b$ [25 and 26]. Therefore, the calculated results of this paper for the other scattering parameters are in good agreement with experimental data. 


\section{Quantum scattering in two dimensions}

Scattering in two dimensions is almost similar to the one dimensional scattering. The timeindependent Schrödinger equation in polar coordinates is considered as follow [15]:

$$
\frac{1}{r} \frac{\partial}{\partial r}\left(r \frac{\partial \psi}{\partial r}\right)+\frac{1}{r^{2}} \frac{\partial^{2} \psi}{\partial \theta^{2}}+\left[k^{2}-U(\vec{r})\right] \psi=0,
$$

Where $k^{2}=2 \mu E / \hbar^{2}$ and $U(\vec{r})=2 \mu V(\vec{r}) / \hbar^{2}$. Here $U(\vec{r})$ is a central potential (depending only on the magnitude of $r$ ).

The scattering amplitude is written in terms of the scattering phase shift $\delta_{m}(k)$ as follow [3]:

$$
f(k, \theta)=\sqrt{\frac{2}{\pi}} \sum_{m=0}^{\infty} \cos (m \theta) \varepsilon_{m} \mathrm{e}^{\mathrm{i} \delta_{m}(k)} \sin \delta_{m}(k)
$$

Where $\varepsilon_{m}=2$ for $m \neq 0$ and $\varepsilon_{0}=1$ for $m=0$. Using the differential scattering cross section $\mathrm{d} \sigma_{2 D} / \mathrm{d} \theta=$ $|f(k, \theta)| / k$, the total cross section, can be obtained as follow [3, 4 and 11]:

$$
\sigma_{2 D}\left(k^{2}\right)=\int_{0}^{2 \pi}\left(d \sigma_{2 D} / d \theta\right)=4 \sum_{0}^{\infty} \varepsilon_{m} \sin ^{2} \delta_{m}(k) .
$$

Where $\theta$ is the scattering angle. The equation for WKB phase shift is mentioned bellow [27]:

$$
\delta_{2 D}^{W K B}(k, m)=m \pi / 2+\int_{r_{0}}^{\infty} d r\left(F^{1 / 2}-k\right)-k r_{0} .
$$

In this equation $k^{2}=2 \mu E / \hbar^{2}$ is the wave number, ${ }^{m \equiv k b^{\prime}}$ where $b^{\prime}$ is the impact parameter and $F$ is defined as follow [27]:

$$
F=k^{2}-U(\vec{r})-\frac{\left(m^{2}-\frac{1}{4}\right)}{r^{2}}
$$

\section{Scattering in two dimensions in the presence of a noncentral potential}

In order to study the neutron- proton scattering in two dimensions, a noncentral two-dimensional potential function with the following form is considered in this paper [28]:

$$
V(r, \theta)=a r^{2}+\frac{b}{r^{2}}+\beta \frac{\cot ^{2} \theta}{r^{2}}+c \quad \beta>0
$$

Where $\mathrm{a}, \mathrm{b}$ and $\mathrm{c}$ are constant values [29 and 30]. The time- independent Schrödinger equation with the potential $V(r, \theta)$ is as follow:

$$
\nabla^{2} \psi(\vec{r})+\frac{2 \mu}{\hbar^{2}}[E-V(r, \theta)] \psi(\vec{r})=0
$$

where $\psi(\vec{r})=\psi(r, \theta, \varphi)=R(r) H(\theta) \phi(\varphi)$. By separating the radial and angular variables into two parts, the equation related to the radial part will be:

$$
\frac{d^{2} U(x)}{d x^{2}}+\frac{1}{2 x} \frac{d U(x)}{d x}+\frac{2 \mu}{\hbar^{2}} \frac{1}{4 x^{2}}\left(E x-a x^{2}-b-\frac{\hbar^{2}}{2 \mu} \gamma\right) U(x)=0
$$

order to solve this equation by NU method [22], the transformations $R(r)=\frac{1}{r} U(r), x=r^{2}$ are applied. Then, the obtained equation is compared with equation (7), the corresponding polynomials in NU technique are achieved as follow: 


$$
\tilde{\tau}(x)=1 \quad \sigma(x)=2 x \quad \sigma^{\prime}(x)=2 \quad \tilde{\sigma}(x)=\frac{2 \mu}{\hbar^{2}}\left(E x-a x^{2}-b-\frac{\hbar^{2}}{2 \mu} \gamma\right)
$$

Substituting these polynomials in equation (13), the function $\pi(x)$ will be:

$$
\pi(x)=\frac{1}{2} \pm \frac{1}{2} \sqrt{\left(\frac{8 \mu}{\hbar^{2}} a\right) x^{2}-\left(\frac{8 \mu}{\hbar^{2}} E+8 k\right) x+\frac{8 \mu}{\hbar^{2}} b+4 \gamma+1}
$$

The constant $k$ can be determined according to the rule that the expression under the square root must be square of a polynomial, therefore:

$$
k_{1,2}=-\frac{\mu}{\hbar^{2}} E \pm \frac{1}{2} \sqrt{\frac{2 \mu}{\hbar^{2}} a \zeta} .
$$

Where $\zeta=4 \gamma+\frac{8 \mu}{\hbar^{2}} b+1$ substituting these two values of $k$ in equation (37), the possible solutions for are $\pi(x)$ :

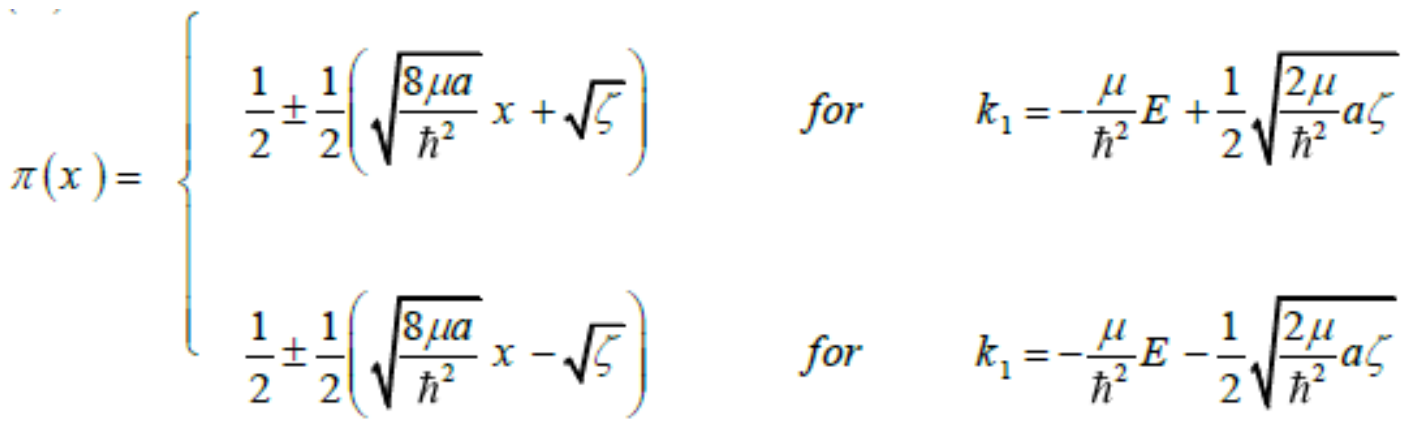

Consequently, $\operatorname{since}^{\tau(x)}$ in equation (15) must have a negative derivative, the most appropriate answers for $\pi(x), k$ and $\tau(x)$ are as follows:

$$
\begin{aligned}
& \pi(x)=\frac{1}{2}-\frac{1}{2}\left(\sqrt{\frac{8 \mu a}{\hbar^{2}}} x+\sqrt{\zeta}\right) \\
& \tau(x)=2-\sqrt{\frac{8 \mu a}{\hbar^{2}}} x+\sqrt{\zeta} \\
& k=-\frac{\mu}{\hbar^{2}} E+\frac{1}{2} \sqrt{\frac{2 \mu}{\hbar^{2}} a \zeta .}
\end{aligned}
$$

Finally, one can immediately determine the energy eigenvalues of the radial part as:

$$
E=\frac{\sqrt{\frac{8 \mu a}{\hbar^{2}}}(-1-2 n)+\sqrt{\frac{2 \mu a}{\hbar^{2}} \zeta}}{2 \mu / \hbar^{2}}
$$

On the other hand, the equation related to the angular part of the time- independent Schrödinger equation will be:

$$
\frac{d^{2} H}{d \theta^{2}}+\cot \theta \frac{d H}{d \theta}+\left[\frac{\hbar^{2}}{2 \mu} \gamma-\frac{2 \mu}{\hbar^{2}} \beta \cot ^{2} \theta-\frac{m^{2}}{\sin ^{2} \theta}\right] H(\theta)=0
$$


Where $m^{2}$ is the separation constant.

By changing the variable $x=\cos \theta$, the equation (44) will become:

$$
\frac{d^{2} H}{d x^{2}}-\frac{2 x}{1-x^{2}} \frac{d H}{d x}+\frac{1}{\left(1-x^{2}\right)^{2}}\left[\frac{\hbar^{2}}{2 \mu} \gamma\left(1-x^{2}\right)-\frac{2 \mu}{\hbar^{2}} \beta x^{2}-m^{2}\right] H(\theta)=0
$$

Comparing this equation with equation (7), the corresponding polynomials will be:

$$
\tilde{\tau}(x)=-2 x \quad \sigma(x)=1-x^{2} \quad \sigma^{\prime}(x)=-2 x \quad \tilde{\sigma}(x)=-\left(\frac{\hbar^{2}}{2 \mu} \gamma+\frac{2 \mu}{\hbar^{2}} \beta\right) x^{2}+\frac{\hbar^{2}}{2 \mu} \gamma-m^{2}
$$

Substituting these polynomials in equation (13), the function $\pi(x)$ will be achieved as follow:

$$
\pi(x)= \pm \sqrt{\left(\frac{\hbar^{2}}{2 \mu} \gamma+\frac{2 \mu}{\hbar^{2}} \beta-k\right) x^{2}-\frac{\hbar^{2}}{2 \mu} \gamma-m^{2}+k}
$$

Considering the special condition for $k$, the following possible solutions for $\pi(x)$ are obtained:

$$
\pi(x)=\left\{\begin{array}{lll} 
\pm \sqrt{\frac{2 \mu}{\hbar^{2}} \beta+m^{2}} & \text { for } & k_{1}=\frac{\hbar^{2}}{2 \mu} \gamma-\frac{2 \mu}{\hbar^{2}} ; \\
\pm \sqrt{\frac{2 \mu}{\hbar^{2}} \beta+m^{2}} x & \text { for } & k_{2}=\frac{\hbar^{2}}{2 \mu} \gamma-m^{2}
\end{array}\right.
$$

Which according to the condition $\tau^{\prime}<0$, the most appropriate answers for $\pi(x)$ and $k$ will be:

$$
\pi(x)=-\sqrt{\frac{2 \mu}{\hbar^{2}} \beta+m^{2}} x \quad \text { for } \quad k_{2}=\frac{\hbar^{2}}{2 \mu} \gamma-m^{2}
$$

Using equation (14), yields the value of $\gamma$ which is important in calculation of the energy eigenvalues.

$$
\gamma=\frac{2 \mu}{\hbar^{2}}\left(n(1-n)+m^{2}+(2 n+1) \sqrt{m^{2}+\frac{2 \mu}{\hbar^{2}} \beta}\right)
$$

\subsection{Scattering cross section, length and amplitude in two dimensions}

Doing numerical calculations at various low energies and using the related equations for scattering in two dimensions mentioned before, the scattering cross section, amplitude and length in the presence of a two-dimensional noncentral potential are obtained. The results are listed in the following table. As mentioned before, these results are reasonably consistent with the experimental data for the scattering cross section [25 and 26].

Table 2: Scattering cross section, length and amplitude in the presence of the noncentral potential mentioned in this paper (with $n=0, l=0, m^{2}=1$ )

\begin{tabular}{|c|c|c|c|c|c|c|c|c|}
\hline$a(\mathrm{fm})$ & $b(\mathrm{fm})$ & $\beta(\mathrm{fm})$ & $b^{\prime}(\mathrm{fm})$ & $\theta$ & $E(\mathrm{MeV})$ & $f(k, \theta)$ & $\sigma_{\text {tot }}(b)$ & $a_{0}(\mathrm{fm})$ \\
\hline 0.15 & 5.50 & 0.02 & 1.25 & 12 & 2.26 & 6.65 & 5.53 & 5.34 \\
\hline 0.34 & 2.48 & 0.05 & 1.80 & 20 & 3.05 & 6.23 & 4.97 & 6.13 \\
\hline
\end{tabular}




\begin{tabular}{|l|l|l|l|l|l|l|l|l|}
\hline 0.30 & 3.99 & 0.06 & 1.80 & 18 & 3.20 & 5.25 & 4.98 & 6.04 \\
\hline 0.37 & 4.95 & 0.06 & 1.80 & 15 & 3.74 & 5.19 & 4.88 & 6.76 \\
\hline 0.38 & 7.25 & 0.02 & 1.25 & 10 & 3.89 & 5.09 & 4.08 & 6.93 \\
\hline
\end{tabular}

It is worth mentioning that the consistency of the results in this paper with the reported experimental observations for scattering cross section indicates the reliability of the suggested method for the other calculated parameters.

\section{Conclusion}

In this paper, a suitable central and an appropriate noncentral potential are considered and the energy eigenvalues of the Schrödinger equation are obtained. Consequently, the Phase shift, scattering cross section, amplitude and length for neutron-proton scattering in one and two dimensions are calculated. Comparison between the calculated results and reported experimental values demonstrates acceptable agreement between them.

\section{References}

[1] L. D. Landau and E. M. Lifshitz, "Quantum Mechanics, Non-Relativistic Theory", 3rd edition, Pergamum, New York, (1977).

[2] V. E Barlette, M. M Leite and S. K Adhikari, "Quantum Scattering in one Dimension”, Eur. J. Phys. 21, 435-440 (2000).

[3] I. R. Lapidus, "Quantum-mechanical scattering in 2 dimensions", Am. J. Phys. 50, 45-47 (1982).

[4] M. J. Moritz and H. Friedrich, "Scattering by a Coulomb field in two dimensions", Am. J. Phys. 66, 274 (1998).

[5] I. R. Lapidus, "Scattering by two-dimensional circular barrier, hard circle, and delta-function ring Potentials", Am. J. Phys. 54, 459-461(1986).

[6] J. J. Sakurai, "Modern Quantum Mechanics”, Addison Wesley, (1994).

[7] A. Agarwal, A. E. Lipstein, and D. Young, "Scattering amplitudes of massive N=2 gauge theories in three dimensions", Phys. Rev. D 89, 045020 (2014)

[8] G. Barton, "Rutherford scattering in 2 dimensions", Am. J. Phys. 51, 420-422 (1983).

[9] P. A. Maurone and T.K. Lim, "More on two-dimensional scattering", Am. J. Phys. 51, 856-857 (1983).

[10] K. S. Krane, "Introductory Nuclear Physics", John Wiley and Sons, New York, (1987).

[11] S. K. Adhikari, "Quantum scattering in two dimensions", Am. J. Phys. 54, 362-367 (1986).

[12] M. Farrokh, M. R. Shojaei and A. A. Rajabi, "Klein-Gordon equation with Hulth'en potential and position-dependent mass", Eur. Phys. J. Plus 128, 14 (2013).

[13] R. G. Newton, "Scattering Theory of Waves and Particles", 2nd Edition, Springer-Verlag, New York, (1982).

[14] H. Feizi, M.R. Shojaei and A. A. Rajabi., "Raising and lowering operators for the DiracWoods-Saxon potential in the presence of spin and pseudospin symmetry", Eur. Phys. J. Plus 127, 41 (2012).

[15] S. K. Adhikari and M. S. Hussein, "Semi-classical scattering in two dimensions", Am. J. Phys. 70, 663 (2008).

[16] S. K. Adhikari, "Variational Principles and the Numerical Solution of Scattering Problems", New York: John Wiley, (1998).

[17] T. Miyazawa, "Boson representations of one-dimensional scattering", J. Phys. A- Math. Gen. 33, 191 (2000).

[18] M. Visser, "Some general bounds for one-dimensional scattering”, Phys. Rev. A. 59, 427 (1999). 
[19] L. V. Chebotarev and A. Tchebotareva, "Flat resonances in one-dimensional quantum scattering”, J. Phys. A 29, 7259 (1996).

[20] Y. Nogami and C. K. Ross, "Scattering from a Nonsymmetric potential in one Dimension as a Coupled-channel Problem", Am. J. Phys. 64, 923-928 (1996).

[21] S. M. Ikhdair and R. Sever, "Approximate Analytical Solutions of the Generalized WoodsSaxon Potentials Including the Spin-Orbit Coupling Term and Spin-Symmetry", Cent. Eur. J. Phys. $8,652,(2010)$.

[22] A. F. Nikiforov and V. B. Uvarov, "Special Functions of Mathematical Physics", Birkhäuser, Boston, (1988).

[23] M. R. Shojaei, A. A. Rajabi, M. Farrokh, N. Zoghi-Foumani, "Energy Levels of Spin-1/2 Particles with Yukawa Interaction”, JMP 5, 773-780 (2014).

[24]A. N. Ikot and I. O. Akpan, "Bound State Solutions of Schrödinger Equation for a more general Woods-Saxon Potential with Arbitrary - state", Chinese Phys. Lett. 29090302 (2012). 1

[25] W. Greiner, "Quantum Mechanics, an introduction", 4th edition, Springer-Verlag, New York, (1989).

[26] H. S. Hans, "Nuclear Physics: Experimental and Theoretical", New Age International, (2001).

[27] M. S. Hussein, Y. T. Chen and F. I. A. Almedia, "The Validity of the Classical Description of Nuclear Scattering”, Am. J. Phys. 52, 650-653, (1984).

[28] S. M. Ikhdair and R. Sever, "Exact Solutions of the D-Dimensional Schrödinger Equation for a Ring-Shaped Pseudoharmonic potential”, Cent. Eur. Phys. 6(3), 685-696 (2008).

[29] M. L. Sage, "The vibrations and rotations of the pseudogaussian oscillator", Chem. Phys. 87, 431-439 (1984).

[30] M. L. Sage and J. Goodisman, "Improving on the conventional presentation of molecular vibrations: advantages of the pseudoharmonic potential and the direct construction of potential energy curves", Am. J. Phys. 53, 350-355 (1985). 